\title{
Analytic Smoothing Effect for the Schrödinger Equation with Long-Range Perturbation
}

\author{
André Martinez ${ }^{1}$, Shu Nakamura ${ }^{2}$, Vania Sordoni ${ }^{1}$
}

December 20, 2004

\begin{abstract}
We study microlocal analytic singularity of solutions to Schrödinger equation with analytic coefficients. Using microlocal weight estimate developped for estimating the phase space tunneling, we prove microlocal smoothing estimates that generalize results by L. Robbiano and C. Zuily. We suppose the Schrödinger operator is a long-range type perturbation of the Laplacian, and we employ positive commutator type estimates to prove the smoothing property.
\end{abstract}

\section{Introduction}

It is well-known that solutions to the Schrödinger equation have infinite propagation speed, and hence we cannot expect propagation of singularity theorems similar to that for the wave equation. Instead, local smoothing effect has been used to study the local smoothness of solutions to Schrödinger equations. The smoothing effects for the Schrödinger equation has been a very rich source of investigations during the last past years: see, e.g., [Ze, Sjl, Yam, Yaj1, HaKa1, Yaj2, GiVe, KPV, KaSa, CKS, KaTa, KaYa, KRY, HaKa2, Wu, RoZu1, RoZu2, KaWa, MRZ, Do1, RoZu3, HaWu, Na2, Na3, Do2]. In particular, Craig, Kappeler and Strauss [CKS] showed that this effect may be considered as a microlocal phenomenon, and this observation inspired series of investigations, both in the $C^{\infty}$-case (in particular $[\mathrm{Wu}, \mathrm{HaWu}])$ and in the analytic case ([RoZu1, RoZu2, RoZu3]). While the papers [Wu, HaWu, RoZu3] address the case of the Laplacian associated to a certain generalization of asymptotically flat metrics (the so-called scattering metrics, defined on a compact manifold with boundary, where the boundary

\footnotetext{
${ }^{1}$ Università di Bologna, Dipartimento di Matematica, Piazza di Porta San Donato 5, 40127 Bologna, Italy. Partly supported by Università di Bologna, Funds for Selected Research Topics and Founds for Agreements with Foreign Universities

${ }^{2}$ Graduate School of Mathematical Science, University of Tokyo, 3-8-1 Komaba, Meguro-ku, Tokyo, Japan 153-8914.
} 
plays the role of the infinity), the papers [RoZu1, RoZu2] concentrate more specifically on the case of asymptotically flat metrics on $\mathbb{R}^{n}$. In all of these five papers, the perturbations are assumed to be of short-range type (in the sense that $\sigma>1$ in Assumption A). Their methods rely on a special notion of wave front set (the quadratic scattering wave font set) for which results of propagation are proved by using constructions of microlocal parametrices. As it is often the case with such a method, construction and the computations are relatively complicated. Nakamura [Na2] gave a simpler proof in the case of asymptotically flat metrics with long-range $C^{\infty}$-class perturbations, using a different notion of wave front set (the homogeneous wave front set) for which the result of propagation is obtained through positive commutator type argument (which is similar to the original proof of the propagation of singularity theorem by Hörmander [Ho]). More precise characterization of the $C^{\infty}$-wave front set of solutions to Schrödinger equations is studied in [Na3] using an Egorov theorem type argument.

The purpose of this paper is to prove a theorem similar to [Na2] for the analytic singularity. The proof is relatively simple, and we recover the results of [RoZu1, RoZu2] without using a construction of parametrix, but rather using microlocal energy estimates in the same spirit as in [Ma2]. We note this method has been employed to give simpler argument to study the analytric wave front set, or, more generally, of the microsupport of solutions of analytic partial differential equations (see, e.g., [Ma2] Chapter 4). In this paper, we apply a generalization of the method to study the analytic homogeneous wave front set and analytic smoothing effects.

It is not completely clear to us whether our notion of wave front set coincides exactly or not with that of [RoZu1, RoZu2], but we will see that the results of these papers can be recovered from our main theorem. Moreover, our argument allows us to generalize these results to the case of long-range perturbations in a sense similar to that of [Na2] (that is, the perturbation is not only assumed to decay more slowly, but the coefficients of the lower order terms are indeed allowed to have some polynomial growth at infinity). In addition, we can also relax the assumptions of analyticity on the coefficients of the operator, in the sense that we assume that they can extended holomorphically to a strip in $\mathbb{C}^{n}$ around $\mathbb{R}^{n}$ (rather than a complex sector as in [RoZu1, RoZu2]).

In the next section we specify our assumptions, introduce the notion of analytic homogeneous wave front set, and state our main results. Section 3 is devoted to a discussion of microlocal exponential weight estimate, in a spirit similar to that of [Ma2], but with a Bargmann transform that involves an additional parameter. In Section 4, we prove our main results, and we give the details on the argument to recover the result of [RoZu2] in Appendix A. The other 3 appendices discuss proof of technical lemmas.

Acknowledgments A.M. would like to thank L. Robbiano and C. Zuily for valuable discussions on the subject and for enlightening explanations on 
their own works. The authors would also like to thank M. Zworski for the interest he has shown for this work and stimulating discussions.

\section{Notations and Main Result}

We consider the analytic wave front set of solutions to Schrödinger equation with variable coefficients. Namely, we set

$$
P=\frac{1}{2} \sum_{j, k=1}^{n} a_{j k}(x) D_{j} D_{k}+\sum_{j=1}^{n} a_{j}(x) D_{j}+a_{0}(x)
$$

on $\mathcal{H}=L^{2}\left(\mathbb{R}^{n}\right)$, where $D_{j}=-i \partial_{x_{j}}$. We suppose the coefficients $\left\{a_{\alpha}(x)\right\}$ satisfy the following assumptions. For $\nu>0$ we denote

$$
\Gamma_{\nu}=\left\{z \in \mathbb{C}^{n}|| \operatorname{Im} z \mid<\nu\right\} .
$$

Assumption A. For each $\alpha, a_{\alpha}(x) \in C^{\infty}\left(\mathbb{R}^{n}\right)$, the $a_{j k}(x)$ 's are real-valued and the matrix $\left(a_{j k}(x)\right)_{1 \leq j, k \leq n}$ is symmetric and positive definite. We set $b_{j}(x)=\operatorname{Re} a_{j}(x), c_{j}(x)=\operatorname{Im} a_{j}(x)$ for $j=0,1, \ldots, n$ and we assume $a_{j k}(x)$, $b_{j}(x), c_{j}(x)$ are extended to holomorphic functions on $\Gamma_{\nu}$ with some $\nu>0$. Moreover, there is $\sigma>0$ such that for all $\beta \in \mathbb{Z}_{+}^{n}$,

$$
\begin{aligned}
& \left|\partial_{z}^{\beta}\left(a_{j k}(z)-\delta_{j k}\right)\right| \leq C_{\beta}\langle z\rangle^{-\sigma-|\beta|}, \quad j, k=1, \ldots, n, \\
& \left|\partial_{z}^{\beta} b_{j}(z)\right| \leq C_{\beta}\langle z\rangle^{1-\sigma-|\beta|}, \quad j=1, \ldots, n, \\
& \left|\partial_{z}^{\beta} c_{j}(z)\right| \leq C_{\beta}\langle z\rangle^{-\sigma-|\beta|}, \quad j=1, \ldots, n \\
& \left|\partial_{z}^{\beta} b_{0}(z)\right| \leq C_{\beta}\langle z\rangle^{2-\sigma-|\beta|}, \\
& \left|\partial_{z}^{\beta} c_{0}(z)\right| \leq C_{\beta}\langle z\rangle^{1-\sigma-|\beta|}
\end{aligned}
$$

for $z \in \Gamma_{\nu}$ with some $C_{\beta}>0$.

We consider the solution $u(t) \in C\left([0, T] ; L^{2}\left(\mathbb{R}^{n}\right)\right)$ to the time-dependent Schrödinger equation:

$$
\left\{\begin{array}{l}
i \frac{\partial u}{\partial t}=P u \quad(t \in(0, T)) \\
\left.u\right|_{t=0}=u_{0}
\end{array}\right.
$$

where $u_{0} \in L^{2}\left(\mathbb{R}^{n}\right)$. We denote the $L^{2}$-norm by $\|\cdot\|$ without subscript.

In order to describe the analytic singularity, we use the flat FBI transform (or the Bargmann transform with parameters, or the Gaussian wave packet transform in physics literature). Let $h, \mu>0$, and we set

$$
T_{h, \mu} u(x, \xi)=c_{h, \mu} \int_{\mathbb{R}^{n}} e^{i(x-y) \cdot \xi / h} e^{-\mu|x-y|^{2} / 2 h} u(y) d y
$$


for $u \in \mathcal{S}\left(\mathbb{R}^{n}\right)$, where $c_{h, \mu}=2^{-n / 2} \mu^{-n / 4}(\pi h)^{-3 n / 4}$. It is well-known that $T_{h, \mu}$ is extended to an isometry from $L^{2}\left(\mathbb{R}^{n}\right)$ to $L^{2}\left(\mathbb{R}^{2 n}\right)$. $T_{h, \mu}$ is also extended to a continuous linear map from $\mathcal{S}^{\prime}\left(\mathbb{R}^{n}\right)$ to $C^{\infty}\left(\mathbb{R}^{2 n}\right)$. The analytic wave front set is defined as follows:

Definition 1. Let $\left(x_{0}, \xi_{0}\right) \in \mathbb{R}^{n} \times\left(\mathbb{R}^{n} \backslash 0\right)$, and let $u \in \mathcal{S}^{\prime}\left(\mathbb{R}^{n}\right)$. Then, $\left(x_{0}, \xi_{0}\right)$ is not in the analytic wave front set of $u$ (i.e., $\left.\left(x_{0}, \xi_{0}\right) \notin W F_{a}(u)\right)$ if there is $\delta, \varepsilon>0$ such that

$$
\left\|T_{h, \mu} u\right\|_{L^{2}\left(B_{\varepsilon}\left(\left(x_{0}, \xi_{0}\right)\right)\right)} \leq C \exp (-\delta / h), \quad \text { for } 0<h \leq 1 .
$$

Here we denote the open ball of radius $r>0$ with the center at $X$ by $B_{r}(X)$ :

$$
B_{r}(X)=\{Y|| X-Y \mid \leq r\},
$$

and $\mu>0$ is arbitrarily fixed.

Remark. $W F_{a}(u)$ is independent of the choice of $\mu>0$ since $T_{h, \mu^{\prime}} T_{h, \mu}^{*}$ is an integral operator on $L^{2}\left(\mathbb{R}^{2 n}\right)$ with a Gaussian kernel. Hence we may fix $\mu=1$. There are equivalent definitions of the analytic wave front set (cf. [Sjs], [Ma2]). We choose this definition because it fits nicely to our argument.

We also use an analogue of the homogeneous wave front set introduced in $[\mathrm{Na} 2]$.

Definition 2. Let $\left(x_{0}, \xi_{0}\right) \in \mathbb{R}^{n} \times\left(\mathbb{R}^{n} \backslash 0\right)$, and let $u \in \mathcal{S}^{\prime}\left(\mathbb{R}^{n}\right)$. $\left(x_{0}, \xi_{0}\right)$ is not in the analytic homogeneous wave front set of $u$ (i.e., $\left(x_{0}, \xi_{0}\right) \notin H W F_{a}(u)$ ) if there is $\delta>0$ and a neighborhood $\Sigma$ of $\left(x_{0}, \xi_{0}\right)$ which is conic with respect to the group of variables $(x, \xi) \in \mathbb{R}^{2 n}$, and such that

$$
\left\|e^{\delta(|x|+|\xi|)} T_{1, \mu} u\right\|_{L^{2}(\Sigma)}<\infty,
$$

where $\mu>0$ is fixed.

Remark. The definition of $H W F_{a}(u)$ is independent of the choice of $\mu$ as well as $W F_{a}(u)$. Thus we may set $\mu=1$, but it is often convenient to use various $\mu>0$. We can unify the notion of $W F_{a}$ and $H W F_{a}$ using the Fourier-Bros-Iagolnitzer transform $\mathbf{T}$ defined by,

$$
\mathbf{T} u(x, \xi):=\int e^{i(x-y) \xi-\frac{\langle\xi\rangle}{\langle x\rangle}(x-y)^{2}} u(y) d y .
$$

This can be seen by using the techniques of analytic microlocal analysis introduced in [Sjs] (in particular the proof of Proposition 6.2).

To state our main result, we recall notations of the Hamiltonian flow generated by the Riemannian metric $\left\{a_{j k}(x)\right\}$. Let

$$
p(x, \xi)=\frac{1}{2} \sum_{j, k=1}^{n} a_{j k}(x) \xi_{j} \xi_{k},
$$


and let $\gamma=\{(y(t), \eta(t)) ; t \in \mathbb{R}\}$ be a corresponding integral curve of the Hamilton flow, that is, a solution of

$$
\dot{y}(t)=\frac{\partial p}{\partial \xi}(y(t), \eta(t)), \quad \dot{\eta}(t)=-\frac{\partial p}{\partial x}(y(t), \eta(t)) .
$$

We say that $\gamma$ is backward nontrapping if $|(y(t), \eta(t))| \rightarrow \infty$ as $t \rightarrow-\infty$. If $\gamma$ is backward nontrapping, it is well-known that the asymptotic momentum,

$$
\eta_{-}=\lim _{t \rightarrow-\infty} \eta(t)
$$

exists, provided Assumption A is satisfied (cf., e.g., [CKS]). Our main result is the following one:

Theorem 2.1. Suppose Assumption A, and suppose $\gamma$ is backward nontrapping. Let $\eta_{-}$be the asymptotic momentum as $t \rightarrow-\infty$. Suppose there exists $t_{0}>0$ such that

$$
\left(-t_{0} \eta_{-}, \eta_{-}\right) \notin H W F_{a}\left(u_{0}\right),
$$

then

$$
\left(\left(t-t_{0}\right) \eta_{-}, \eta_{-}\right) \notin H W F_{a}(u(t)) \quad \text { for } \quad 0<t<\min \left(t_{0}, T\right),
$$

and moreover, if $t_{0}<T$, then,

$$
\gamma \cap W F_{a}(u(t))=\emptyset,
$$

for all $t$ close enough to $t_{0}$.

Remark. Actually, we prove a stronger result than (2.6). Namely, the statement holds true also for the uniform analytic wave front set as defined in [RoZu1, RoZu2].

Now we can recover and generalize the following two results of Robbiano and Zuily (we note $\sigma>1$ and decaying condition on lower-order coefficients are assumed in [RoZu1, RoZu2]):

Corollary 2.2. Assume Assumption $A$ and $\gamma$ is backward nontrapping. Moreover assume that $e^{\delta_{0}|x|} u_{0} \in L^{2}\left(\Sigma_{\varepsilon_{0}}\right)$ for some $\delta_{0}, \varepsilon_{0}>0$, where

$$
\Sigma_{\varepsilon_{0}}:=\bigcup_{s \leq 0}\left\{x|| x-y(s) \mid \leq \varepsilon_{0}(1+|s|)\right\} .
$$

Then we have

$$
\gamma \cap W F_{a}(u(t))=\emptyset
$$

for any $t>0$.

Indeed, in this case (2.4) is satisfied for any $t_{0}>0$, since $|\xi|=O(|x|)$ on any small enough conic neighborhood of $\left(-t_{0} \eta_{-}, \eta_{-}\right)$. 
Corollary 2.3. Assume Assumption $A$ and $\gamma$ is backward nontrapping. Moreover, assume that $u_{0}(x)=a(x) e^{i \psi(x)}$ where $a \in L^{2}\left(\mathbb{R}^{n}\right) ; \psi$ is realvalued and analytic on $\mathbb{R}^{n}$; and for some $m \geq 1$, a and $\psi$ admit holomorphic extensions on a set of the form

$$
\tilde{\Sigma}_{\varepsilon_{0}}:=\left\{\left.x \in \mathbb{C}^{n}\left|\operatorname{Re} x \in \Sigma_{\varepsilon_{0}},\right| \operatorname{Im} x\left|<\delta_{0}\right| \operatorname{Re} x\right|^{\min (m-1,1)},|x|>R\right\}
$$

and verify the following estimates on this set:

$$
|\psi(x)|=O\left(|x|^{m}\right), \quad|a(x)|=O\left(|x|^{M}\right)
$$

with some $M \geq 1$. In the case $m=1$, assume also $|\nabla \psi(x)|=O(1)$ on the same set. Moreover, assume there exist $t_{0}>0$ and a positive constant $C$ such that,

$$
\left|\nabla \psi\left(-\lambda t_{0} \eta_{-}\right)-\lambda \eta_{-}\right| \geq \frac{\lambda^{m-1}}{C}
$$

for all $\lambda>0$ sufficiently large. Then,

$$
\gamma \cap W F_{a}(u(t))=\emptyset
$$

for any $t>0$.

In this case (2.4) is satisfied with this value of $t_{0}$ (see Appendix A).

\section{$3 \quad$ Exponential weight estimates}

Here we discuss a key estimate of the proof of Theorem 2.1. We assume $0<\sigma \leq 1$ without loss of generality and, for simplicity, we write $T=T_{h, \mu}$. Let $\psi(x, \xi) \in C_{0}^{\infty}\left(\mathbb{R}^{2 n}\right)$ be an $(h, \mu)$-dependent function such that there exists $C_{1}>1$ such that

$$
\operatorname{supp}[\psi] \subset\left\{(x, \xi)\left|\frac{1}{C_{1}} \leq\right| \xi \mid \leq C_{1}, \frac{1}{C_{1} \mu} \leq\langle x\rangle \leq \frac{C_{1}}{\mu}\right\}
$$

and that for any multi-indeces $\alpha, \beta \in \mathbb{Z}_{+}^{n}$,

$$
\left|\partial_{x}^{\alpha} \partial_{\xi}^{\beta} \psi(x, \xi)\right| \leq C_{\alpha \beta} \mu^{|\alpha|}, \quad x, \xi \in \mathbb{R}^{n},
$$

uniformly with respect to $h, \mu \in(0,1]$. We also suppose

$$
\sup _{x, \xi}\left|\partial_{x} \psi(x, \xi)\right|<\nu, \quad \sup _{x, \xi}\left|\partial_{\xi} \psi(x, \xi)\right|<\nu
$$

for any $h, \mu \in(0,1]$. We let $f \in C_{0}^{\infty}\left(\mathbb{R}^{2 n}\right)$ such that

$$
\operatorname{supp}[f] \subset\left\{(x, \xi)\left|\frac{1}{C_{2}} \leq\right| \xi \mid \leq C_{2}, \frac{1}{C_{2} \mu} \leq\langle x\rangle \leq \frac{C_{2}}{\mu}\right\}
$$


with $C_{2}>C_{1}$ and that

$$
f=1 \quad \text { on } \operatorname{supp}[\psi] .
$$

Moreover, we suppose that for any $\alpha, \beta \in \mathbb{Z}_{+}^{n}$,

$$
\left|\partial_{x}^{\alpha} \partial_{\xi}^{\beta} f(x, \xi)\right| \leq C_{\alpha \beta}^{\prime} \mu^{|\alpha|}, \quad x, \xi \in \mathbb{R}^{n},
$$

and that $f \geq 0, \sqrt{f} \in C_{0}^{\infty}\left(\mathbb{R}^{2 n}\right)$ and $\sqrt{f}$ satisfies the same estimates. We denote

$$
\begin{aligned}
\tilde{p}(x, \xi) & =h^{-2} \frac{1}{2} \sum_{j, k=1}^{n} a_{j k}(x) \xi_{j} \xi_{k}+h^{-1} \sum_{j=1}^{n} a_{j}(x) \xi_{j}+a_{0}(x), \\
\tilde{p}_{\psi}(x, \xi) & =\tilde{p}\left(x-\partial_{\mu} \psi(x, \xi), \xi+i \mu \partial_{\mu} \psi(x, \xi)\right),
\end{aligned}
$$

where

$$
\partial_{\mu}=\mu^{-1} \partial_{x}+i \partial_{\xi}
$$

Then we have:

Theorem 3.1. Let $\psi$ and $f$ as above and suppose $0<h / \mu \leq d$ with some $d>0$. Then there exists $C>0$ such that

$$
\begin{aligned}
& \quad\left|\left\langle e^{\psi / h} T u, f e^{\psi / h} T P u\right\rangle-\left\langle e^{\psi / h} T u, f \tilde{p}_{\psi} e^{\psi / h} T u\right\rangle\right| \\
& \quad \leq C\left(h^{-1} \mu^{1+\sigma}+\mu^{\sigma}+h \mu^{\sigma-1}\right)\left\|\sqrt{f} e^{\psi / h} T u\right\|^{2}+C\left(h^{-1} \mu+\mu^{\sigma}+h \mu^{\sigma-1}\right)\|u\|^{2}
\end{aligned}
$$

for any $u \in L^{2}\left(\mathbb{R}^{n}\right)$.

By elementary computations, we can estimate $\operatorname{Im} \tilde{p}_{\psi}$ as follows:

Lemma 3.2. There exists $C>0$ such that

$$
\left|\operatorname{Im} \tilde{p}_{\psi}(x, \xi)-h^{-2} H_{p} \psi(x, \xi)\right| \leq C\left(h^{-2} \mu^{2}+h^{-1} \mu^{\sigma}+\mu^{\sigma-1}\right),
$$

where $H_{p} \psi=\frac{\partial p}{\partial \xi} \cdot \frac{\partial \psi}{\partial x}-\frac{\partial p}{\partial x} \cdot \frac{\partial \psi}{\partial \xi}$, and $p$ is the principal symbol of $H$ defined by $(2.3)$.

Combining these, we have the following key estimate in the proof of Theorem 2.1:

Corollary 3.3. Under the same assumption as Theorem 3.1, there exists $C>0$ such that

$$
\begin{aligned}
& \left|\operatorname{Im}\left\langle e^{\psi / h} T u, f e^{\psi / h} T P u\right\rangle-\left\langle e^{\psi / h} T u, f\left(h^{-2} H_{p} \psi\right) e^{\psi / h} T u\right\rangle\right| \\
& \leq C\left(h^{-2} \mu^{2}+h^{-1} \mu^{\sigma}+\mu^{\sigma-1}\right)\left\|\sqrt{f} e^{\psi / h} T u\right\|^{2} \\
& \quad+C\left(h^{-1} \mu+\mu^{\sigma}+h \mu^{\sigma-1}\right)\|u\|^{2} .
\end{aligned}
$$


We prove Theorem 3.1 in the remainder of this section. We follow the argument of [Ma2], [Na1], with an additional parameter $\mu$ and different symbol classes. We note $\mu$ may be considered as a scaling parameter with respect to $x$, whereas $h$ is a scaling parameter with respect to $\xi$. We sometimes use the abbreviation: $T_{\psi}=e^{\psi / h} T$. In the following, we always assume

$$
0<h / \mu \leq d
$$

with some constant $d>0$. We denote

$$
\begin{aligned}
& p_{2}(x, \xi)=\frac{1}{2} \sum_{j, k=1}^{n}\left(a_{j k}(x)-\delta_{j k}\right) \xi_{j} \xi_{k}, \\
& p_{1}(x, \xi)=\sum_{j=1}^{n} a_{j}(x) \xi_{j}-\frac{1}{2 i} \sum_{j, k=1}^{n} \partial_{x_{j}} a_{j k}(x) \xi_{k}, \\
& p_{0}(x, \xi)=p_{0}(x)=a_{0}(x)-\frac{1}{2 i} \sum_{j=1}^{n} \partial_{x_{j}} a_{j}(x)-\frac{1}{8} \sum_{j, k=1}^{n} \partial_{x_{j}} \partial_{x_{k}} a_{j k}(x) .
\end{aligned}
$$

Then we have

$$
P=\frac{1}{2} h^{-2}\left(h D_{x}\right)^{2}+h^{-2} p_{2}^{W}\left(x, h D_{x}\right)+h^{-1} p_{1}^{W}\left(x, h D_{x}\right)+p_{0}(x),
$$

where $a^{W}\left(x, h D_{x}\right)$ denotes the Weyl-Hörmander quantization of $a$ :

$$
a^{W}\left(x, h D_{x}\right) u(x)=(2 \pi h)^{-n} \iint e^{(x-y) \cdot \xi / h} a\left(\frac{x+y}{2}, \xi\right) u(y) d y d \xi
$$

for $u \in \mathcal{S}\left(\mathbb{R}^{n}\right)$ (cf. [Ho] Section 18.5). Note $p_{1}, p_{0}$ contain quantization error terms.

We set

$$
q_{j}\left(x, \xi, x^{*}, \xi^{*}\right)=p_{j}\left(x-\xi^{*}, x^{*}\right), \quad x, \xi, x^{*}, \xi^{*} \in \mathbb{R}^{n}, j=0,1,2 .
$$

As in [Ma1], we have

$$
T P_{j}=Q_{j} T,
$$

where $P_{j}=p_{j}^{W}\left(x, h D_{x}\right)$ and $Q_{j}=q_{j}^{W}\left(x, \xi, h D_{x}, h D_{\xi}\right)$. We denote

$$
\Phi\left(x, \xi, x^{*}, \xi^{*}\right)=\sqrt{1+|x|^{2} /\left\langle\xi^{*}\right\rangle^{2}} \quad \Psi\left(x, \xi, x^{*}, \xi^{*}\right)=\sqrt{1+|\xi|^{2} /\left\langle\xi-x^{*}\right\rangle^{2}},
$$

and let $\tilde{g}$ be a metric on $\mathbb{R}^{4 n}$ defined by

$$
\tilde{g}=\frac{d x^{2}}{\Phi^{2}}+\frac{d \xi^{2}}{\Psi^{2}}+\frac{d x^{* 2}}{\Psi^{2}}+\frac{d \xi^{* 2}}{\Phi^{2}} .
$$

We use the $S(m, g)$ symbol class notation of Hörmander [Ho] Section 18.5. In particular, $\operatorname{OPS}(m, g)$ is the space of pseudodifferential operators with thier symbol in $S(m, g)$. 
The next two lemmas are fundamental in the pseudodifferential operator calculus on $\operatorname{Ran}\left[e^{\psi / h} T\right]$. They imply that polynomials of $\left\langle h D_{x}-\xi\right\rangle$ and $\left\langle h D_{\xi}\right\rangle$ are uniformly bounded as quadratic forms on $\operatorname{Ran}\left[e^{\psi / h} T\right]$ (the reader will find their proof in Appendix B). We denote by $g_{0}$ the flat metric on $\mathbb{R}^{4 n}$, i.e., $g_{0}=d x^{2}+d \xi^{2}+d x^{* 2}+d \xi^{* 2}$.

Lemma 3.4. Suppose $Q \in O P S\left(\left\langle\xi-x^{*}\right\rangle^{m}\left\langle\xi^{*}\right\rangle^{\ell}, g_{0}\right)$ with some $m, \ell \in \mathbb{R}$. Then there exists $C>0$ such that

$$
\left|\left\langle e^{\psi / h} T u, Q e^{\psi / h} T u\right\rangle\right| \leq C\left\|e^{\psi / h} T u\right\|^{2}
$$

for $u \in \mathcal{S}\left(\mathbb{R}^{n}\right)$.

Lemma 3.5. Suppose $Q \in O P S\left(\langle\xi\rangle^{a}\langle x\rangle^{b}\left\langle\xi-x^{*}\right\rangle^{m}\left\langle\xi^{*}\right\rangle^{\ell}, \tilde{g}\right)$ with some $a, b, m, \ell \in$ $\mathbb{R}$. Then, for any $N>0$, there exists $C>0$ such that

$$
\left|\left\langle e^{\psi / h} T u, f Q e^{\psi / h} T u\right\rangle\right| \leq C\left(\mu^{-b}\left\|\sqrt{f} e^{\psi / h} T u\right\|^{2}+\mu^{-b}\|u\|^{2}\right)
$$

for $u \in \mathcal{S}\left(\mathbb{R}^{n}\right)$.

Given the above two lemmas, it is convenient to consider the symbols $q_{j}$ 's in the following symbol classes.

Lemma 3.6. For $j=0,1,2$, one has,

$$
q_{j} \in S\left(m_{j}, \tilde{g}\right),
$$

with $m_{j}=\langle\xi\rangle^{j}\langle x\rangle^{2-j-\sigma}\left(\left\langle\xi-x^{*}\right\rangle^{j}\left\langle\xi^{*}\right\rangle^{|2-j-\sigma|}\right)$.

Proof. We recall

$$
p_{j} \in S\left(\langle\xi\rangle^{j}\langle x\rangle^{2-j-\sigma}, g\right),
$$

where $g=d x^{2} /\langle x\rangle^{2}+d \xi^{2} /\langle\xi\rangle^{2}$. Hence, by the definition of $q_{j}$, we have

$$
q_{j} \in S\left(\left\langle x^{*}\right\rangle^{j}\left\langle x-\xi^{*}\right\rangle^{2-j-\sigma}, \frac{d x^{2}}{\left\langle x-\xi^{*}\right\rangle^{2}}+\frac{d \xi^{2}}{\left\langle x^{*}\right\rangle^{2}}+\frac{d x^{* 2}}{\left\langle x^{*}\right\rangle^{2}}+\frac{d \xi^{* 2}}{\left\langle x-\xi^{*}\right\rangle^{2}}\right)
$$

for $j=0,1,2$. On the other hand, it is easy to see

$$
\begin{aligned}
& \max \left(1, C^{-1}\langle\xi\rangle\left\langle\xi-x^{*}\right\rangle^{-1}\right) \leq\left\langle x^{*}\right\rangle \leq C\langle\xi\rangle\left\langle\xi-x^{*}\right\rangle, \\
& \max \left(1, C^{-1}\langle x\rangle\left\langle\xi^{*}\right\rangle^{-1}\right) \leq\left\langle x-\xi^{*}\right\rangle \leq C\langle x\rangle\left\langle\xi^{*}\right\rangle
\end{aligned}
$$

with some $C>0$. Combining these, we conclude the assertion.

Thanks to Assumption A and (3.1), we have the standard result,

$$
R_{j}:=e^{\psi / h} Q_{j} e^{-\psi / h}-Q_{j \psi} \in O P S\left(h^{2} \Phi^{-2} \Psi^{-2} m_{j}, \tilde{g}\right)
$$


and thus

$$
R_{j} \in O P S\left(h^{2}\langle\xi\rangle^{j-2}\langle x\rangle^{-\sigma-j}\left(\left\langle\xi-x^{*}\right\rangle^{j+2}\left\langle\xi^{*}\right\rangle^{|\sigma-2+j|+2}\right), \tilde{g}\right),
$$

where

$q_{j \psi}\left(x, \xi, x^{*}, \xi^{*}\right)=q_{j}\left(x, \xi, x^{*}+i \partial_{x} \psi, \xi^{*}+i \partial_{\xi} \psi\right)=p_{j}\left(x-\xi^{*}-i \partial_{\xi} \psi, x^{*}+i \partial_{x} \psi\right)$, and $Q_{j \psi}=q_{j \psi}^{W}\left(x, \xi, h D_{x}, h D_{\xi}\right)$.

Applying Lemma 3.5 to $R_{j}$, we have

$$
\left|\left\langle e^{\psi / h} T u, f R_{j} e^{\psi / h} T u\right\rangle\right| \leq C h^{2} \mu^{\sigma+j}\left(\left\|\sqrt{f} e^{\psi / h} T u\right\|^{2}+\|u\|^{2}\right) .
$$

It remains only to estimate $\left\langle e^{\psi / h} T u, f Q_{j \psi} e^{\psi / h} T u\right\rangle$ for $j=0,1,2$. We denote

$$
p_{j \psi}(x, \xi)=q_{j \psi}\left(x, \xi, \xi-\mu \partial_{\xi} \psi, \mu^{-1} \partial_{\xi} \psi\right)=p_{j}\left(x-\partial_{\mu} \psi, \xi+i \mu \partial_{\mu} \psi\right)
$$

for $j=0,1,2$.

Lemma 3.7. There exists $C>0$ such that

(i)

$$
\begin{aligned}
\mid\left\langle e^{\psi / h} T u, f e^{\psi / h}\left\{\frac{1}{2}\left(h D_{x}\right)^{2}-\frac{1}{2}(\xi\right.\right. & \left.\left.\left.+i \mu \partial_{\mu} \psi(x, \xi)\right)^{2}+\frac{n}{4} \mu h\right\} T u\right\rangle \mid \\
& \leq C h \mu\left(\mu\left\|\sqrt{f} e^{\psi / h} T u\right\|^{2}+\|u\|^{2}\right),
\end{aligned}
$$

(ii)

$$
\begin{aligned}
\mid\left\langle e^{\psi / h} T u, f\left\{Q_{j \psi}-p_{j \psi}(x, \xi)\right.\right. & \} e^{\psi / h} T u\right\rangle \mid \\
& \leq C h \mu^{j-1+\sigma}\left(\left\|\sqrt{f} e^{\psi / h} T u\right\|^{2}+\|u\|^{2}\right),
\end{aligned}
$$

for $u \in \mathcal{S}\left(\mathbb{R}^{n}\right), j=0,1,2$, and $h, \mu \in(0,1]$ such that $h / \mu \leq d$.

Proof. (i) By Lemma C.1, we have

$$
\begin{aligned}
& \left\langle e^{\psi / h} T u, f e^{\psi / h}\left(h D_{x}\right)^{2} T u\right\rangle \\
& =\left\langle e^{\psi / h} T u,\left[\left(\xi+i \mu \partial_{\mu} \psi+\frac{i}{2} h \mu \partial_{\mu}\right)^{2} f(x, \xi)\right] e^{\psi / h} T u\right\rangle \\
& =\left\langle e^{\psi / h} T u,\left[\left(\xi+i \mu \partial_{\mu} \psi\right)^{2} f-n \frac{h \mu}{2} f\right.\right. \\
& \left.\left.\quad \quad+i h \mu\left(\xi+i \mu \partial_{\mu} \psi\right) \partial_{\mu} f-\frac{1}{2} h \mu^{2}\left(\partial_{\mu}^{2} \psi\right) f-\frac{1}{4} h^{2} \mu^{2} \partial_{\mu}^{2} f\right] e^{\psi / h} T u\right\rangle,
\end{aligned}
$$

and the claim follows immediately.

(ii) We consider the case $j=2$ only. The claim for the other cases can be shown similarly. We write

$$
\begin{aligned}
& q_{2 \psi}\left(x, \xi, x^{*}, \xi^{*}\right)-q_{2 \psi}\left(x, \xi, \xi-\mu \partial_{\xi} \psi, \mu^{-1} \partial_{\xi} \psi\right) \\
& =q_{2 \psi}^{(1)}\left(x, \xi, x^{*}, \xi^{*}\right)\left(x^{*}-\xi+\mu \partial_{\xi} \psi\right)+q_{2 \psi}^{(2)}\left(x, \xi, x^{*}, \xi^{*}\right)\left(\xi^{*}-\mu^{-1} \partial_{\xi} \psi\right),
\end{aligned}
$$


where

$$
\begin{aligned}
& q_{2 \psi}^{(1)}\left(x, \xi, x^{*}, \xi^{*}\right) \\
& \quad=\int_{0}^{1} \frac{\partial q_{2 \psi}}{\partial x^{*}}\left(x, \xi, s x^{*}+(1-s)\left(\xi-\mu \partial_{\xi} \psi\right), s \xi^{*}+(1-s) \mu^{-1} \partial_{x} \psi\right) d s, \\
& q_{2 \psi}^{(2)}\left(x, \xi, x^{*}, \xi^{*}\right) \\
& \quad=\int_{0}^{1} \frac{\partial q_{2 \psi}}{\partial \xi^{*}}\left(x, \xi, s x^{*}+(1-s)\left(\xi-\mu \partial_{\xi} \psi\right), s \xi^{*}+(1-s) \mu^{-1} \partial_{x} \psi\right) d s .
\end{aligned}
$$

It is easy to check

$$
\begin{aligned}
& q_{2 \psi}^{(1)} \in S\left(\langle\xi\rangle\langle x\rangle^{-\sigma}\left(\left\langle\xi-x^{*}\right\rangle^{3}\left\langle\xi^{*}\right\rangle^{\sigma}\right), \tilde{g}\right), \\
& q_{2 \psi}^{(2)} \in S\left(\langle\xi\rangle^{2}\langle x\rangle^{-1-\sigma}\left(\left\langle\xi-x^{*}\right\rangle^{2}\left\langle\xi^{*}\right\rangle^{1+\sigma}\right), \tilde{g}\right) .
\end{aligned}
$$

We denote $Q_{2 \psi}^{(j)}=q_{2 \psi}^{(j) W}\left(x, \xi, h D_{x}, h D_{\xi}\right)$. Then by the symbol calculus, we have

$$
\begin{aligned}
R^{(2)}:=Q_{2 \psi}-q_{2 \psi}\left(x, \xi, \xi-\mu \partial_{\xi} \psi, \mu^{-1} \partial_{x} \psi\right) & \\
& -\frac{1}{2}\left\{A Q_{2 \psi}^{(1)}+Q_{2 \psi}^{(1)} A+B Q_{2 \psi}^{(2)}+Q_{2 \psi}^{(2)} B\right\} \\
& \in O P S\left(h^{2}\langle x\rangle^{-2-\sigma}\left(\left\langle\xi-x^{*}\right\rangle^{4}\left\langle\xi^{*}\right\rangle^{\sigma+2}\right), \tilde{g}\right),
\end{aligned}
$$

where $A=h D_{x}-\xi+\mu \partial_{\mu} \psi(x, \xi)$ and $B=h D_{\xi}-\mu^{-1} \partial_{x} \psi(x, \xi)$. Then, using the equation (B.2), we compute

$$
\begin{array}{rl}
T_{\psi}^{*} & f\left\{A Q_{2 \psi}^{(1)}+Q_{2 \psi}^{(1)} A+B Q_{2 \psi}^{(2)}+Q_{2 \psi}^{(2)} B\right\} T_{\psi} \\
= & T_{\psi}^{*} f\left\{i \mu\left[B, Q_{2 \psi}^{(1)}\right]+(i \mu)^{-1}\left[A, Q_{2 \psi}^{(2)}\right]\right\} T_{\psi} \\
& +T_{\psi}^{*}\left\{([f, A]+i \mu[B, f]) Q_{2 \psi}^{(1)}+\left([f, B]+(i \mu)^{-1}[A, f]\right) Q_{2 \psi}^{(2)}\right\} T_{\psi} \\
= & : T_{\psi}^{*} L_{1} T_{\psi}+T_{\psi}^{*} L_{2} T_{\psi} .
\end{array}
$$

We note

$$
\begin{aligned}
& {\left[B, Q_{2 \psi}^{(1)}\right] \in O P S\left(h\langle x\rangle^{-\sigma}\left(\left\langle\xi-x^{*}\right\rangle^{4}\left\langle\xi^{*}\right\rangle^{\sigma}\right), \tilde{g}\right),} \\
& {\left[A, Q_{2 \psi}^{(2)}\right] \in O P S\left(h\langle\xi\rangle^{2}\langle x\rangle^{-2-\sigma}\left(\left\langle\xi-x^{*}\right\rangle^{3}\left\langle\xi^{*}\right\rangle^{\sigma+4}\right), \tilde{g}\right) .}
\end{aligned}
$$

Hence, by applying Lemma 3.5, we have

$$
\left|\left\langle e^{\psi / h} T u, L_{1} e^{\psi / h} T u\right\rangle\right| \leq C h \mu^{1+\sigma}\left(\left\|\sqrt{f} e^{\psi / h} T u\right\|^{2}+\|u\|^{2}\right) .
$$

On the other hand, by applying Lemma B.1, we also have

$$
\left|\left\langle e^{\psi / h} T u, L_{2} e^{\psi / h} T u\right\rangle\right| \leq C h \mu^{1+\sigma}\left(\left\|\sqrt{f} e^{\psi / h} T u\right\|^{2}+\|u\|^{2}\right) .
$$

Finally, by (3.6) and Lemma 3.5, we learn

$$
\left|\left\langle e^{\psi / h} T u, f R^{(2)} e^{\psi / h} T u\right\rangle\right| \leq C h^{2} \mu^{2+\sigma}\left(\left\|\sqrt{f} e^{\psi / h} T u\right\|^{2}+\|u\|^{2}\right)
$$

Combining these we conclude the assertion (ii) for $j=2$. 
Now we recall (3.2). Combining Lemma 3.7 with (3.5), we conclude Theorem 3.1.

\section{Proof of Theorem 2.1}

We suppose $\gamma=\{(y(t), \eta(t)) \mid t \in \mathbb{R}\}$ is backward nontrapping, $t_{0}>$ 0 , and let $\eta_{-}=\lim _{t \rightarrow-\infty} \eta(t)$ as in the assumptions of Theorem 2.1. We suppose $\left(-t_{0} \eta_{-}, \eta_{-}\right) \notin H W F_{a}\left(u_{0}\right)$, hence we can find a conic neighborhood of $\left(-t_{0} \eta_{-}, \eta_{-}\right): \Gamma \subset \mathbb{R}^{2 n}$ and $\delta_{1}>0$ such that

$$
\left\|e^{\delta_{1}(|x|+|\xi|)} T_{1,1} u_{0}\right\|_{L^{2}(\Gamma)}<\infty .
$$

For $\left(x^{\prime}, \xi^{\prime}\right) \in \mathbb{R}^{2 n}$ and $a_{1}, a_{2}>0$, we denote a neighborhood of $\left(x^{\prime}, \xi^{\prime}\right)$ of size $\left(a_{1}, a_{2}\right)$ by

$$
B\left(x^{\prime}, \xi^{\prime} ; a_{1}, a_{2}\right)=\left\{(x, \xi) \in \mathbb{R}^{2 n}|| x-x^{\prime}\left|<a_{1},\right| \xi-\xi^{\prime} \mid<a_{2}\right\} .
$$

Then, for sufficiently small $\delta \in\left(0,\left|\eta_{-}\right|\right)$, we have

$$
\left\|T_{1,1} u_{0}\right\|_{L^{2}\left(B\left(-h^{-1} t_{0} \eta_{-}, h^{-1} \eta_{-} ; h^{-1} \delta t_{0}, h^{-1} \delta\right)\right)} \leq C e^{-\delta / h}
$$

for $h \in(0,1]$. By a change of integration variables, it is equivalent to

$$
\left\|T_{h, h} u_{0}\right\|_{L^{2}\left(B\left(-h^{-1} t_{0} \eta_{-}, \eta_{-} ; h^{-1} \delta t_{0}, \delta\right)\right)} \leq C e^{-\delta / h} .
$$

We use the following weight function. Let $\chi \in C_{0}^{\infty}\left(\mathbb{R}_{+}\right)$such that $\chi(r)=1$ if $r \leq 1 / 2, \chi(r)=0$ if $r \geq 1$, and that $\chi^{\prime}(r) \leq 0$ for $r>0$. We set $\delta_{1}=\delta / 4$ and,

$$
\varphi(t, x, \xi):=\chi\left(\frac{|x-t \xi|}{\delta_{1}|t|}\right) \chi\left(\frac{\left|\xi-\eta_{-}\right|}{\delta_{1}}\right), \quad x, \xi \in \mathbb{R}^{n}, t<0 .
$$

Since $\left(\partial_{t}+H_{\xi^{2} / 2}\right)|x-t \xi|=0, H_{p}=H_{\xi^{2} / 2}+O\left(\langle x\rangle^{-\sigma}|\xi|\right) \partial_{x}+O\left(\langle x\rangle^{-\sigma-1}|\xi|^{2}\right) \partial_{\xi}$, and $|x| \geq \delta|t| / 2$ while $|\xi|=O(1)$ on the support of $\varphi$, we immediately obtain,

$$
\frac{\partial \varphi}{\partial t}+H_{p} \varphi=\frac{|x-t \xi|}{\delta_{1}|t|^{2}} \chi^{\prime}\left(\frac{|x-t \xi|}{\delta_{1}|t|}\right) \chi\left(\frac{\left|\xi-\eta_{-}\right|}{\delta_{1}}\right)+O\left(|t|^{-1-\sigma}\right),
$$

and thus,

$$
\frac{\partial \varphi}{\partial t}+H_{p} \varphi \leq C|t|^{-1-\sigma},
$$

for some constant $C>0$. Now we set

$$
\psi(t, x, \xi)=\delta \varphi\left(h^{-1} t, x, \xi\right),
$$

and we note,

$$
\left.\operatorname{supp}[\psi(t, \cdot, \cdot)] \subset B\left(h^{-1} t \eta_{-}, \eta_{-} ; h^{-1} \delta|t| / 2, \delta / 2\right)\right) .
$$


Hence, we have

$$
\left\|e^{\psi(t, x, \xi) / h} T_{h, h} u_{0}\right\| \leq C<\infty
$$

for $t \in\left[-t_{0}-\varepsilon_{1},-t_{0}+\varepsilon_{1}\right]$ with sufficiently small $\varepsilon_{1}>0$. We then set

$$
\mu(t)=\frac{-t_{0}}{t} h
$$

for $t<0$. We note $\mu(t)$ is monotone increasing and $\mu(t) \geq h$ for $t \in$ $\left[-t_{0}, 0\right)$. It is elementary computation to confirm that $\psi(t, x, \xi)$ satisfies the conditions of Section 3, since $\langle x\rangle^{-1}=O\left(h|t|^{-1}\right)=O(\mu)$ on the support of $\psi(t, \cdot, \cdot)$.

Then, we choose $f \in C^{\infty}\left(\mathbb{R}_{-} ; C_{0}^{\infty}\left(\mathbb{R}^{2 n}\right)\right)$ of the form,

$$
f(t, x, \xi)=\chi_{1}(|\mu(t) x|) \chi_{1}(|\xi|),
$$

where $\chi_{1} \in C_{0}^{\infty}\left((2 A)^{-1} ; 2 A\right), \chi_{1}=1$ on $\left[A^{-1}, A\right]$, with $A>0$ sufficiently large in order to have $f=1$ on the support of $\psi$. In particular, $f$ satisfies to the conditions of Section 3.

Now, we set

$$
F(t)=\left\|\sqrt{f(t, \cdot, \cdot)} e^{\psi(t, \cdot \cdot) / h} T_{h, \mu(t)} u\left(t+t_{0}\right)\right\|^{2}
$$

for $t \in\left[-t_{0}, 0\right)$, and we are find a differential inequality satisfied by $F(t)$. Setting $\tilde{u}(t)=u\left(t+t_{0}\right)$, and writing $T$ instead of $T_{h, \mu(t)}$, we compute,

$$
\begin{aligned}
\frac{d}{d t} F(t)= & \left\langle e^{\psi / h} T(-i P) \tilde{u}, f e^{\psi / h} T \tilde{u}\right\rangle+\left\langle e^{\psi / h} T \tilde{u}, f e^{\psi / h} T(-i P) \tilde{u}\right\rangle \\
& +\left\langle e^{\psi / h} \tilde{u}, 2 h^{-1} f\left(\frac{\partial}{\partial t} \psi\right) e^{\psi / h} T \tilde{u}\right\rangle \\
& +\left\langle e^{\psi / h}\left[\frac{\partial}{\partial t}, T\right] \tilde{u}, f e^{\psi / h} T \tilde{u}\right\rangle+\left\langle e^{\psi / h} T \tilde{u}, f e^{\psi / h}\left[\frac{\partial}{\partial t}, T\right] \tilde{u}\right\rangle \\
& +\left\langle e^{\psi / h} T \tilde{u},\left(\frac{\partial f}{\partial t}\right) e^{\psi / h} T \tilde{u}\right\rangle \\
= & F_{1}+F_{2}+F_{3}+F_{4} .
\end{aligned}
$$

At first, we have

$$
\begin{aligned}
F_{1} & =2 \operatorname{Im}\left[\left\langle e^{\psi / h} T \tilde{u}, f e^{\psi / h} T P \tilde{u}\right\rangle\right] \\
& =2\left\langle e^{\psi / h} T \tilde{u}, f\left(h^{-2} H_{p} \psi\right) e^{\psi / h} T \tilde{u}\right\rangle+r(t),
\end{aligned}
$$

where $r(t)$ is estimated by Corollary 3.3. Hence we learn

$$
\begin{aligned}
F_{1}+F_{2} & =2 h^{-2}\left\langle e^{\psi / h} T \tilde{u}, f\left(h \frac{\partial}{\partial t} \psi+H_{p} \psi\right) e^{\psi / h} T \tilde{u}\right\rangle+r(t) \\
& \leq C h^{-1+\sigma}|t|^{-1-\sigma} F(t)+r(t)
\end{aligned}
$$

using the inequality (4.1).

Lemma 4.1. There exists $C>0$ such that

$$
F_{3}(t) \leq C\left\{h^{-1} \mu^{\prime}(t) F(t)+\mu^{\prime}(t)\|\tilde{u}\|^{2}\right\} .
$$


Proof. We note

$$
\begin{aligned}
{\left[\frac{\partial}{\partial t}, T\right] \tilde{u} } & =c_{h, \mu} \int\left(-\frac{\mu^{\prime}(t)}{2 h}\right)|x-y|^{2} e^{i(x-y) \cdot \xi / h-\mu|x-y|^{2} / 2 h} \tilde{u}(y) d y \\
& =(2 h)^{-1} \mu^{\prime}(t) \int h^{2} \triangle_{\xi} e^{i(x-y) \cdot \xi / h-\mu|x-y|^{2} / 2 h} \tilde{u}(y) d y \\
& =\frac{1}{2} h \mu^{\prime}(t) \triangle_{\xi} T \tilde{u} .
\end{aligned}
$$

Hence we have

$$
\begin{aligned}
F_{3}= & \frac{1}{2} h \mu^{\prime}(t)\left\langle T \tilde{u},\left(\triangle_{\xi} f e^{2 \psi / h}+f e^{\psi / h} \triangle_{\xi}\right) T \tilde{u}\right\rangle \\
= & h \mu^{\prime}(t)\left\langle T \tilde{u},\left\{\sqrt{f} e^{\psi / h} \triangle_{\xi} \sqrt{f} e^{\psi / h}+\left|\nabla_{\xi}\left(\sqrt{f} e^{\psi / h}\right)\right|^{2}\right\} T \tilde{u}\right\rangle \\
\leq & h\left\{h^{-2}\left\langle\sqrt{f} e^{\psi / h} T \tilde{u},\left|\nabla_{\xi} \psi\right|^{2} \sqrt{f} e^{\psi / h} T \tilde{u}\right\rangle\right. \\
& \left.\quad \quad\left\langle T \tilde{u},\left(2 \nabla_{\xi} \sqrt{f} \cdot h^{-1} \nabla_{\xi} \psi+\left|\nabla_{\xi} \sqrt{f}\right|^{2}\right) T \tilde{u}\right\rangle\right\} \\
\leq & C h^{-1} \mu^{\prime}(t)\left\|\sqrt{f} e^{\psi / h} T \tilde{u}\right\|^{2}+C \mu^{\prime}(t)\|\tilde{u}\|^{2},
\end{aligned}
$$

since $\nabla_{\xi} \sqrt{f}$ is supported away from $\operatorname{supp}[\psi]$.

For the last term, we easily have

$$
F_{4}=\left\langle T \tilde{u},\left(\frac{\partial f}{\partial t}\right) T \tilde{u}\right\rangle=O\left(|t|^{-1}\right) .
$$

We note

$$
\mu^{\prime}(t)=t_{0} h t^{-2}=O\left(h|t|^{-2}\right) .
$$

Combining Lemma 4.1 with (4.3) and (4.4), we obtain the following estimate:

Lemma 4.2. There exists $C>0$ such that

$$
\frac{d}{d t} F(t) \leq A(t) F(t)+B(t)\|\tilde{u}\|^{2}
$$

where

$$
A(t)=C\left(h^{-1+\sigma}|t|^{-1-\sigma}+|t|^{-2}\right), \quad B(t)=C|t|^{-1} .
$$

By Gronwall's inequality, we have

$$
\begin{aligned}
F(t) & \leq e^{\int_{-t_{0}}^{t} A(s) d s}\left(F\left(-t_{0}\right)+\int_{-t_{0}}^{t} e^{-\int_{-t_{0}}^{s} A(\tau) d \tau} B(s)\|\tilde{u}\|^{2} d s\right) \\
& \leq e^{\int_{-t_{0}}^{t} A(s) d s}\left(F\left(-t_{0}\right)+\int_{-t_{0}}^{t} B(s) d s \cdot\|\tilde{u}\|^{2}\right) .
\end{aligned}
$$

for $t<0$. For each $t \in\left(-t_{0}, 0\right)$, this immediately implies

$$
F(t)=\left\|\sqrt{f} e^{\psi(t, x, \xi) / h} T_{h,\left|t_{0} / t\right| h} \tilde{u}(t)\right\|^{2}=O\left(e^{C(t) h^{-1+\sigma}}\right)
$$


as $h \rightarrow 0$, with some $C(t)>0$. Since

$$
\psi(t, x, \xi)=\delta \quad \text { on } \quad B\left(h^{-1} t \eta_{-}, \eta_{-} ; h^{-1} \delta_{1}|t| / 4, \delta_{1} / 4\right)
$$

if $h$ is sufficiently small, this implies $\left(t \eta_{-}, \eta_{-}\right) \notin H W F_{a}(\tilde{u}(t))$. This proves the first claim of Themrem 2.1.

Moreover, by elementary computation, we obtain

$$
\int_{-t_{0}}^{-R h} A(t) d t \leq C^{\prime} h^{-1} R^{-\sigma}
$$

for $R \geq 1$. We fix $R>0$ so that

$$
0<C^{\prime} R^{-\sigma} \leq \delta / 2
$$

and hence

$$
\int_{-t_{0}}^{-R h} A(t) d t \leq \delta h^{-1} / 2 \quad \text { as } h \rightarrow 0 .
$$

Similarly, we have

$$
\int_{-t_{0}}^{-R h} B(t) d t=O(|\log h|) \quad \text { as } h \rightarrow 0 .
$$

Combining these estimates, we learn $F(-R h)=O\left(e^{\delta / h}\right)$ as $h \rightarrow 0$, and hence,

$$
e^{-\delta / h} F(-R h)=\left\|\sqrt{f} e^{(\psi(-R h, x, \xi)-\delta / 2) / h} T_{h, \mu(-R h)} \tilde{u}(-R h)\right\|^{2} \leq C<\infty .
$$

We recall $\mu(-R h)=t_{0} / R$ and

$$
\psi(-R h, x, \xi)=\delta \varphi(-R, x, \xi)=\delta \chi\left(\frac{|x+R \xi|}{\delta_{1} R}\right) \chi\left(\frac{\left|\xi-\eta_{-}\right|}{\delta_{1}}\right) .
$$

By standard estimates on the flow, we also know that $\left|-R \eta_{-}-y(-R)\right|=$ $O\left(R^{1-\sigma}\right)$ and $\left|\eta_{-}-\eta(-R)\right|=O\left(R^{-\sigma}\right)$. Thus, increasing $R$ if necessary, we obtain

$$
\left\|T_{h, t_{0} / R} \tilde{u}(-R h)\right\|_{L^{2}\left(B\left(\gamma(-R) ; \delta_{1} R / 8, \delta_{1} / 8\right)\right)}^{2} \leq C e^{-\delta / h} .
$$

Moreover, in the above argument, we may replace $-t_{0}$ by $t$ in a small neighborhood of $-t_{0}$, e.g., $t \in\left[-t_{0}-\varepsilon_{1},-t_{0}+\varepsilon_{1}\right]$ with some $\varepsilon_{1}>0$. Then, we learn

$$
\left\|T_{h, t_{0} / R} \tilde{u}(s-R h)\right\|_{\left.L^{2}\left(\gamma(-R) ; \delta_{1} R / 8, \delta_{1} / 8\right)\right)}^{2} \leq C e^{-\delta / h}
$$

uniformly for $s \in\left[-\varepsilon_{1}, \varepsilon_{1}\right]$. In particular, this implies $\gamma(-R) \notin W F_{a}(\tilde{u}(s))$ for $s \in\left[-\varepsilon_{1} / 2, \varepsilon_{1} / 2\right]$. Then, we use the following variation of the propagation of the microsupport theorem: 
Lemma 4.3. Let $u(t)$ be a solution to the Schrödinger equation for $t$ in a neighborhood of 0 . Then, if the following condition:

$$
\exists \delta, \varepsilon>0 \text { such that }\left\|T_{h, 1} u(h t)\right\|_{L^{2}(B(\gamma(t) ; \delta, \delta))}=O\left(e^{-\varepsilon / h}\right) \text { as } h \rightarrow 0
$$

holds for some $t$, it holds for all $t \in \mathbb{R}$.

This lemma is an immediate consequence of [Ma2], Chapter 4, Exercise 9 , after a scaling of the variable $t: t \mapsto h t$. We give a concise proof in Appendix D for completeness. Now the estimate (4.5) implies that for any $t \in \mathbb{R}, s \in\left[-\varepsilon_{0}, \varepsilon_{0}\right]$, there is $\delta, \varepsilon>0$ such that

$$
\left\|T_{h, 1} \tilde{u}(s-h R+h t)\right\|_{L^{2}(B(\gamma(t-R) ; \delta, \delta))}=O\left(e^{-\varepsilon / h}\right) .
$$

In particular, setting $t^{\prime}=t-R$ and $s^{\prime}=s+h t^{\prime}$, we learn $\gamma\left(t^{\prime}\right) \notin W F_{a}\left(\tilde{u}\left(s^{\prime}\right)\right)$ for any $t^{\prime} \in \mathbb{R}$ and $s^{\prime} \in\left[-\varepsilon_{0} / 2, \varepsilon_{0} / 2\right]$. This completes the proof of Theorem 2.1.

\section{A Proof of Corollary 2.3}

It is enough to show that $T_{1,1} u_{0}=O\left(e^{-\delta\langle\xi\rangle}\right)$ for some $\delta>0$, uniformly for $(x, \xi)$ in a conic neighborhood of $\left(-t_{0} \eta_{-}, \eta_{-}\right)$, where

$$
T_{1,1} u_{0}(x, \xi)=c \int e^{i(x-y) \cdot \xi+i \psi(y)-|x-y|^{2} / 2} a(y) d y,
$$

(with $c:=2^{-n / 2} \pi^{-3 n / 4}$ ).

We first assume $m \geq 2$. For $\lambda \geq 1$, we set $\left(x_{\lambda}, \xi_{\lambda}\right):=\left(-\lambda t_{0} \eta_{-}, \lambda \eta_{-}\right)$ and $\eta_{\lambda}:=\nabla \psi\left(x_{\lambda}\right)$. Then, for $y \in \mathbb{C}^{n}$ such that $\left|y-x_{\lambda}\right| \leq 2 \varepsilon \lambda$ (with $\varepsilon>0$ small enough), using the analyticity of $\psi$, standard Cauchy estimates, and the fact that $\psi$ is real on the real, by a second-order Taylor expansion at $x_{\lambda}$, we see that,

$$
\operatorname{Im} \psi(y)=\operatorname{Im}\left(y \cdot \eta_{\lambda}\right)+O\left(\varepsilon \lambda^{m-1}|t \operatorname{Im} y|\right)
$$

uniformly for $\lambda \geq 1$ and $\varepsilon>0$ small enough.

Now, we apply the following change of contour of integration in (A.1):

$$
\mathbb{R}^{n} \ni y \mapsto z:=y-i \delta \lambda \frac{\xi-\eta_{\lambda}}{\left|\xi-\eta_{\lambda}\right|} \chi\left(\lambda^{-1}\left|y-x_{\lambda}\right|\right),
$$

(where $\chi \in C_{0}^{\infty}([0,2 \varepsilon)), \chi=1$ on $[0, \varepsilon]$, and $\varepsilon \geq \delta>0$ are small enough). On this contour, we have,

$$
\begin{aligned}
\operatorname{Re} & \left(i(x-z) \xi+i \psi(z)-|x-z|^{2} / 2\right) \\
\quad & =\operatorname{Im} z \cdot\left(\xi-\eta_{\lambda}\right)-|x-y|^{2} / 2+|\operatorname{Im} z|^{2} / 2+O\left(\varepsilon \lambda^{m-1}|\operatorname{Im} z|\right) \\
& =-\delta \lambda\left|\xi-\eta_{\lambda}\right| \chi-|x-y|^{2} / 2+\delta^{2} \lambda^{2} \chi^{2}+O\left(\varepsilon \delta \lambda^{m} \chi\right) .
\end{aligned}
$$


In particular, using (2.7), we obtain for $\lambda^{-1} \xi$ close enough to $\eta_{-}$,

$$
\begin{aligned}
& \operatorname{Re}\left(i(x-z) \cdot \xi+i \psi(z)-|x-z|^{2} / 2\right) \\
& \quad \leq-\delta \lambda^{m} \chi /(2 C)-|x-y|^{2} / 2+\delta^{2} \lambda^{2} \chi^{2}+O\left(\varepsilon \delta \lambda^{m} \chi\right)
\end{aligned}
$$

and thus, by choosing first $\varepsilon$ small enough, and then $\delta$ small enough (and since $m \geq 2$ ),

$$
\operatorname{Re}\left(i(x-z) \cdot \xi+i \psi(z)-|x-z|^{2} / 2\right) \leq-\delta \lambda^{m} \chi /(4 C)-|x-y|^{2} / 2 .
$$

As a consequence, for $\lambda^{-1}\left|x-x_{\lambda}\right| \leq \varepsilon / 2$, we obtain,

$$
\begin{aligned}
& \left|T_{1,1} u_{0}(x, \xi)\right| \\
& =O\left(\int_{\left|y-x_{\lambda}\right| \leq \varepsilon \lambda}\langle y\rangle^{M} e^{-\delta \lambda^{m} /(4 C)} d y+\int_{\left|y-x_{\lambda}\right| \geq \varepsilon \lambda}\langle y\rangle^{M} e^{-\varepsilon^{2} \lambda^{2} / 16-|x-y|^{2} / 4} d y\right) \\
& =O\left(\lambda^{M+n} e^{-\delta \lambda^{m} /(4 C)}+\lambda^{M} e^{-\varepsilon \lambda^{2} / 16}\right)
\end{aligned}
$$

and the result for $m=2$ follows.

In the case $m=1$, we observe that $\nabla \psi(y)=O(1)$ and Hess $\psi(y)=O(1)$ uniformly on $\left\{y \in \mathbb{C}^{n}|| \operatorname{Re} y-x_{\lambda}|\leq \varepsilon \lambda,| \operatorname{Im} y \mid \leq \varepsilon\right\}$, and thus, by a firstorder Taylor expansion, $\operatorname{Im} \psi(y)=O((1+\varepsilon \lambda)|\operatorname{Im} y|)$ on this set. Therefore, applying in (A.1) the following change of contour of integration:

$$
\mathbb{R}^{n} \ni y \mapsto z:=y-i \delta \frac{\xi}{|\xi|} \chi\left(\lambda^{-1}\left|y-x_{\lambda}\right|\right),
$$

(with $\delta \leq \varepsilon$ and $\chi$ as before), this time we obtain,

$\operatorname{Re}\left(i(x-z) \cdot \xi+i \psi(z)-|x-z|^{2} / 2\right)=-\delta|\xi| \chi-|x-y|^{2} / 2+O(1+\varepsilon \delta \lambda \chi)$

and thus, for $\lambda^{-1}(x, \xi)$ close enough to $\left(-t_{0} \eta_{-}, \eta_{-}\right)$, we obtain as before,

$$
\left|T_{1,1} u_{0}(x, \xi)\right|=O\left(\lambda^{M+n} e^{-\delta \lambda / 2}+\lambda^{M} e^{-\varepsilon \lambda^{2} / 16}\right),
$$

and the result follows.

Remark. Actually, in the case $m \geq 2$, we have indeed proved that $T_{1} u_{0}=$ $O\left(e^{-\delta\langle\xi\rangle^{2}}\right)$ for some $\delta>0$, uniformly for $(x, \xi)$ in a $\mathbb{R}^{2 n}$-conic neighborhood of $\left(-t_{0} \eta_{-}, \eta_{-}\right)$.

\section{B Proof of Lemmas 3.4 and 3.5}

Proof of Lemma 3.4. We prove the lemma by induction in $m$ and $\ell$. The claim is obvious if $m=\ell=0$. We suppose the claim holds for $(m, \ell)$ and prove the claim for $(m+1, \ell)$ and $(m, \ell+1)$. We write

$$
A=h D_{x}-\xi+\mu \partial_{\xi} \psi(x, \xi), \quad B=h D_{\xi}-\mu^{-1} \partial_{x} \psi(x, \xi) .
$$


We recall (cf. [Ma2], [Na1]):

$$
(A-i \mu B) e^{\psi / h} T u=0 \quad \text { for } \quad u \in \mathcal{S}\left(\mathbb{R}^{n}\right),
$$

which can be shown by straightforward computaion. If $Q \in O P S(\langle\xi-$ $\left.\left.x^{*}\right\rangle^{m+1}\left\langle\xi^{*}\right\rangle^{\ell}, g_{0}\right)$ we write

$$
Q=\tilde{Q}(A+i), \quad \tilde{Q}=Q(A+i)^{-1} \in O P S\left(\left\langle\xi-x^{*}\right\rangle^{m}\left\langle\xi^{*}\right\rangle^{\ell}, g_{0}\right) .
$$

Then we have

$$
\begin{aligned}
T_{\psi}^{*} \tilde{Q} A T_{\psi} & =\frac{1}{2} T_{\psi}^{*}\{\tilde{Q} A+A \tilde{Q}+[\tilde{Q}, A]\} T_{\psi} \\
& =\frac{i \mu}{2} T_{\psi}^{*}[\tilde{Q}, B] T_{\psi}+\frac{1}{2} T_{\psi}^{*}[\tilde{Q}, A] T_{\psi}
\end{aligned}
$$

It is easy to check $[\tilde{Q}, A],[\tilde{Q}, B] \in O P S\left(h\left\langle\xi-x^{*}\right\rangle^{m}\left\langle\xi^{*}\right\rangle^{\ell}, g_{0}\right)$. By the induction hypotesis, we learn $\left\langle T_{\psi} u, \tilde{Q} A T_{\psi} u\right\rangle \leq C\left\|T_{\psi} u\right\|^{2}$.

Similarly, if $Q \in O P S\left(\left\langle\xi-x^{*}\right\rangle^{m}\left\langle\xi^{*}\right\rangle^{\ell+1}, g_{0}\right)$, then we write

$$
Q=\tilde{Q}(B+i), \quad \tilde{Q}=Q(B+i)^{-1} \in O P S\left(\left\langle\xi-x^{*}\right\rangle^{m}\left\langle\xi^{*}\right\rangle^{\ell}, g_{0}\right),
$$

and we compute

$$
T_{\psi}^{*} \tilde{Q} B T_{\psi}=\frac{1}{2 i \mu} T_{\psi}^{*}[\tilde{Q}, A] T_{\psi}+\frac{1}{2} T_{\psi}^{*}[\tilde{Q}, B] T_{\psi} .
$$

We note $[\tilde{Q}, A] \in O P S\left(h\left\langle\xi-x^{*}\right\rangle^{m}\left\langle\xi^{*}\right\rangle^{\ell}, g_{0}\right)$, and since $h / \mu \leq d$, we have

$$
\left\langle T_{\psi} u, \tilde{Q} B T_{\psi} u\right\rangle \leq C(h / \mu+1)\left\|T_{\psi} u\right\|^{2} \leq C^{\prime}\left\|T_{\psi} u\right\|^{2}
$$

again using the induction hypothesis.

Proof of Lemma 3.5. Since $\langle\xi\rangle=O(1)$ and $\langle x\rangle=O\left(\mu^{-1}\right)$ on the support of $f$, we may assume $a=b=0$ without loss of generality. Analogously to the above lemma, we prove the assertion by induction in $m$ and $\ell$. At first, we suppose $Q \in S(1, \tilde{g})$. Then

$$
\left\langle T_{\psi} u, f Q T_{\psi} u\right\rangle=\left\langle\sqrt{f} T_{\psi} u, Q \sqrt{f} T_{\psi} u\right\rangle+\left\langle T_{\psi} u,[Q, \sqrt{f}] \sqrt{f} T_{\psi} u\right\rangle .
$$

The first term in the right hand side is bounded by $C\left\|\sqrt{f} T_{\psi} u\right\|^{2}$ since $Q$ is bounded in $L^{2}\left(\mathbb{R}^{2 n}\right)$. The second term is estimated using the following lemma:

Lemma B.1. Let $Q \in O P S\left(\langle\xi\rangle^{a}\langle x\rangle^{b}\left\langle\xi-x^{*}\right\rangle^{m}\left\langle\xi^{*}\right\rangle^{\ell}, \tilde{g}\right)$, and suppose the symbol of $Q$ has an asymptotic expansion supported in $\operatorname{supp}[\nabla f]$. Then for any $N>0$, there exists $C>0$ such that

$$
\left|\left\langle e^{\psi / h} T u, Q e^{\psi / h} T u\right\rangle\right| \leq C\left(h^{N} \mu^{N}\left\|\sqrt{f} e^{\psi / h} T u\right\|^{2}+\mu^{-b}\|u\|^{2}\right)
$$

for $u \in \mathcal{S}\left(\mathbb{R}^{n}\right)$. 
We postpone the proof of Lemma B.1, and proceed with the proof of Lemma 3.5. Since the symbol of $[Q, \sqrt{f}]$ has an asymptotic expansion supported in $\operatorname{supp}[\nabla f]$, we can apply Lemma B.1 to learn

$$
\left|\left\langle T_{\psi} u,[Q, \sqrt{f}] T_{\psi} u\right\rangle\right| \leq C\left(h^{N} \mu^{N}\left\|\sqrt{f} T_{\psi} u\right\|^{2}+\|u\|^{2}\right) .
$$

Thus the claim holds if $m=\ell=0$.

Now suppose $Q \in O P S\left(\left\langle\xi-x^{*}\right\rangle^{m+1}\left\langle\xi^{*}\right\rangle^{\ell}, \tilde{g}\right)$. Denoting $\tilde{Q}=Q(A+i)^{-1} \in$ $O P S\left(\left\langle\xi-x^{*}\right\rangle^{m}\left\langle\xi^{*}\right\rangle^{\ell}, \tilde{g}\right)$, we have

$$
\begin{aligned}
T_{\psi}^{*} f Q T_{\psi}= & T_{\psi}^{*} f \tilde{Q} A T_{\psi}+i T_{\psi}^{*} f \tilde{Q} T_{\psi} \\
= & \frac{i \mu}{2} T_{\psi}^{*} f[\tilde{Q}, B] T_{\psi}+\frac{1}{2} T_{\psi}^{*} f[\tilde{Q}, A] T_{\psi}+\frac{1}{2} T_{\psi}^{*}[f, A] \tilde{Q} T_{\psi} \\
& \quad+\frac{i \mu}{2} T_{\psi}^{*}[B, f] \tilde{Q} T_{\psi}+i T_{\psi}^{*} f \tilde{Q} T_{\psi} .
\end{aligned}
$$

The first, the second and the last terms in the right hand side are estimated by the induction hypotesis, and the third and the fourth terms are estimated by Lemma B.1 to obtain the assertion for $(m+1, \ell)$. The argument for the case $Q \in O P S\left(\left\langle\xi-x^{*}\right\rangle^{m}\left\langle\xi^{*}\right\rangle^{\ell+1}, \tilde{g}\right)$ is similar, and we omit the detail.

Proof of Lemma B.1. By the assumption on $f$, we can find $\tilde{f} \in C_{0}^{\infty}\left(\mathbb{R}^{2 n}\right)$ such that (i) $\tilde{f}=1$ on $\operatorname{supp}[\psi]$; (ii) $f=1$ on $\operatorname{supp}[\tilde{f}]$; (iii) for any $\alpha, \beta \in \mathbb{Z}_{+}^{n}$, $\left|\partial_{x}^{\alpha} \partial_{\xi}^{\beta} \tilde{f}(x, \xi)\right| \leq C_{\alpha \beta} \mu^{|\alpha|}\left(x, \xi \in \mathbb{R}^{n}\right)$. We write

$$
Q=(1-\tilde{f}) Q(1-\tilde{f})+\{\tilde{f} Q+Q \tilde{f}-\tilde{f} Q \tilde{f}\}=Q_{1}+Q_{2} .
$$

The symbol of $Q_{2}$ has vanishing asymptotic expansion since $\operatorname{supp}[\nabla f] \cap$ $\operatorname{supp}[\tilde{f}]=\emptyset$. In particular, since $\mu^{-N}\langle x\rangle^{-N} \tilde{f}$ is uniformly bounded together with all its derivatives, we can deduce,

$$
Q_{2} \in O P S\left(h^{N} \mu^{N-b}\left\langle\xi-x^{*}\right\rangle^{m+N}\left\langle\xi^{*}\right\rangle^{\ell+N}, \tilde{g}\right)
$$

for any $N$. Hence, by Lemma 3.4, we have

$$
\begin{aligned}
\mid\left\langle e^{\psi / h} T u\right. & \left., Q_{2} e^{\psi / h} T u\right\rangle \mid \leq C h^{N}\left\langle\langle x\rangle^{b-N} e^{\psi / h} T u, e^{\psi / h} T u\right\rangle \\
& \leq C h^{N} \mu^{N-b}\left\{\left\langle e^{\psi / h} T u, f e^{\psi / h} T u\right\rangle+\left\langle e^{\psi / h} T u,(1-f) e^{\psi / h} T u\right\rangle\right\} \\
& \leq C h^{N} \mu^{N-b}\left(\left\|\sqrt{f} e^{\psi / h} T u\right\|^{2}+\|u\|^{2}\right) .
\end{aligned}
$$

On the other hand, we note $Q_{1} \in O P S\left(\left(\mu^{-b}+h^{N}\right)\left\langle\xi-x^{*}\right\rangle^{m+|a|}\left\langle\xi^{*}\right\rangle^{\ell+|b|}, \tilde{g}\right)$ for all $N$ (where $h^{N}$ comes from the remainder term of the asymptotic expansion of the symbol), and $e^{\psi / h} Q_{1} e^{\psi / h}=Q_{1}$. Hence, for $h \leq d \mu$, we have

$$
\left|\left\langle e^{\psi / h} T u, Q_{1} e^{\psi / h} T u\right\rangle\right|=\left|\left\langle T u, Q_{1} T u\right\rangle\right| \leq C \mu^{-b}\|u\|^{2}
$$

again by Lemma 3.4. Combining these, we obtain the assertion. 


\section{Exponential weight formula for differential op- erators}

Here we prove an exponential weight formula for differential operators, which was introduced in Introduction of [Ma1]. We reproduce the proof for the reader's convenience.

Lemma C.1. Suppose $f \in C_{0}^{\infty}\left(\mathbb{R}^{2 n}\right)$, and we write $T=T_{h, \mu}$. Then for any $\alpha \in \mathbb{Z}_{+}^{n}$,

$$
\left\langle e^{\psi / h} T v, f e^{\psi / h} T\left(h D_{x}\right)^{\alpha} u\right\rangle=\frac{1}{2}\left\langle e^{\psi / h} T v,\left[\left(\xi+i \mu \partial_{\mu} \psi+\frac{i}{2} h \mu \partial_{\mu}\right)^{\alpha} f\right] e^{\psi / h} T u\right\rangle
$$

for $u, v \in \mathcal{S}\left(\mathbb{R}^{n}\right)$.

Proof. At first, we note

$$
\begin{aligned}
e^{\psi / h} T\left(h D_{x}\right) & =e^{\psi / h}\left(h D_{x}\right) T=\left(h D_{x}+i \partial_{x} \psi\right) e^{\psi / h} T \\
& =\left(A+\xi+i \mu \partial_{\mu} \psi\right) e^{\psi / h} T
\end{aligned}
$$

where $A$ is defined in (B.1). On the other hand, by virtue of (B.2), we have

$$
\begin{aligned}
\left\langle e^{\psi / h}\right. & \left.T v, f A e^{\psi / h} T u\right\rangle \\
& =\frac{1}{2}\left\langle e^{\psi / h} T v,(A f+f A) e^{\psi / h} T u\right\rangle+\frac{1}{2}\left\langle e^{\psi / h} T v,[f, A] e^{\psi / h} T u\right\rangle \\
& =\frac{1}{2}\left\langle e^{\psi / h} T v,(-i \mu B f+i \mu f B) e^{\psi / h} T u\right\rangle+\frac{1}{2}\left\langle e^{\psi / h} T v,[f, A] e^{\psi / h} T u\right\rangle \\
& =\frac{1}{2}\left\langle e^{\psi / h} T v,[f, A+i \mu B] e^{\psi / h} T u\right\rangle \\
& =\frac{1}{2}\left\langle e^{\psi / h} T v,\left(i h \mu \partial_{\mu} f\right) e^{\psi / h} T u\right\rangle .
\end{aligned}
$$

Combining these, we learn

$$
\left\langle e^{\psi / h} T v, f e^{\psi / h} T\left(h D_{x}\right) u\right\rangle=\left\langle e^{\psi / h} T v,\left[\left(\xi+i \mu \partial_{\mu} \psi+\frac{i}{2} h \mu \partial_{\mu}\right) f\right] e^{\psi / h} T u\right\rangle .
$$

Iterating this procedure, we conclude the assertion.

\section{Proof of Lemma 4.3}

Let $t_{0}<t_{1}$, and we suppose (4.6) is satisfied for $t=t_{0}$. We choose $\varphi \in C_{0}^{\infty}\left(B\left(\gamma\left(t_{0}\right) ; \delta, \delta\right)\right)$ such that $\varphi=1$ on $B\left(\gamma\left(t_{0}\right) ; \delta / 2, \delta / 2\right)$. By the assumption, we have

$$
\left\|e^{\varepsilon \varphi(x, \xi) / h} T_{h, 1} u\left(h t_{0}\right)\right\| \leq C<\infty \quad \text { as } \quad h \rightarrow 0,
$$

for sufficiently small $\varepsilon>0$. We then set

$$
\psi(t, x, \xi)=\varphi\left(\exp \left(t_{0}-t\right) H_{p}(x, \xi)\right)
$$


for $t \in\left[t_{0}, t_{1}\right], x, \xi \in \mathbb{R}^{n}$. We also choose $f \in C_{0}^{\infty}\left(\mathbb{R}^{2 n}\right)$ so that

$$
f(x, \xi)=1 \quad \text { on } \quad \bigcup_{t_{0} \leq t \leq t_{1}} \operatorname{supp}[\psi(t, \cdot, \cdot)] .
$$

Then we apply Theorem 3.1 with $\mu=1$ to obtain

$$
\begin{aligned}
& \mid\left\langle e^{\varepsilon \psi(t) / h} T_{h, 1} u(h t), f e^{\varepsilon \psi(t) / h} T_{h, 1} P u(h t)\right\rangle \\
& -\left\langle e^{\varepsilon \psi(t) / \hbar} T_{h, 1} u(h t), \tilde{p}_{\varepsilon \psi} e^{\varepsilon \psi(t) / h} T_{h, 1} u(h t)\right\rangle \mid \\
& \leq C h^{-1}\left(\left\|e^{\varepsilon \psi(t) / h} T_{h, 1} u(h t)\right\|^{2}+\|u(h t)\|^{2}\right)
\end{aligned}
$$

uniformly for $t \in\left[t_{0}, t_{1}\right], \varepsilon \in(0,1]$. Analogously to Lemma 3.2, we have

$$
\left|\operatorname{Im} \tilde{p}_{\varepsilon \psi}(x, \xi)-h^{-2} \varepsilon H_{p} \psi\right| \leq C h^{-2} \varepsilon^{2} .
$$

We note $\frac{\partial}{\partial t} \psi+H_{p} \psi=0$. Hence, analogously to Lemma 4.1, we have

$$
h^{-1} \frac{d}{d t} F(t) \leq C\left(h^{-1}+h^{-2} \varepsilon^{2}\right) F(t)+C h^{-1}\|u(h t)\|^{2}
$$

for $t \in\left[t_{0}, t_{1}\right]$, where $F(t)=\left\|\sqrt{f} e^{\varepsilon \psi(t) / h} T_{h, 1} u(h t)\right\|^{2}$. Hence we learn

$$
F\left(t_{1}\right) \leq C_{1} \varepsilon^{-C_{2}} e^{C_{3} \varepsilon^{2} / h}
$$

where $C_{1}, C_{2}, C_{3}>0$ are independent of $h$ and $\varepsilon$. Thus we obtain

$$
\left\|T_{h, 1} u\left(h t_{1}\right)\right\|_{L^{2}\left(B\left(\gamma\left(t_{1}\right) ; \delta^{\prime}, \delta^{\prime}\right)\right)}^{2} \leq C_{1} \varepsilon^{-C_{2}} e^{-\left(2 \varepsilon-C_{3} \varepsilon^{2}\right) / h}
$$

with sufficiently small $\delta^{\prime}>0$. By choosing $\varepsilon$ sufficiently small, we conclude (4.6) for $t=t_{1}$. Proof for the case $t_{1}<t_{0}$ is similar, and we omit the detail.

\section{References}

[CKS] Craig, W., Kappeler, T., Strauss, W., Microlocal dispersive smoothing for the Schrödinger equation, Comm. Pures Appl. Math. 48 (1996), 769-860.

[Do1] Doi, S., Smoothing effects for Schrödinger evolution equation and global behavior of geodesic flow, Math. Ann. 318 (2000), 355-389.

[Do2] Doi, S., Singularities of solutions of Schrödinger equations for perturbed harmonic oscillators, Hyperbolic problems and related topics, Grad. Ser. Anal. 185-199, Int. Press, Somerville, MA, 2003. 
[GiVe] Ginibre, J., Velo, G., Smoothing properties and retarded estimates for some dispersive evolution equations, Comm. Math. Phys. 144 (1992), 163-188.

[HaWu] Hassel, A., Wunsch, J., The Schrödinger propagator for scattering metrics, Preprint 2003.

[HaKa1] Hayashi, N., Kato, K., Analyticity and smoothing effect for the Schrödinger equation, Ann. Inst. H. Poincaré, Phys. Théo. 52 (1990), 163-173.

[HaKa2] Hayashi, N., Kato, K., Analyticity in time and Smoothing effect of solutions to nonlinear Schrödinger equations, Comm. Math. Phys. 184 (1997), 273-300.

[Ho] Hörmander, L.: The Analysis of Linear Partial Differential Operators, Vol III, Springer Verlag, 1983-1985.

[KaWa] Kajitani, K., Wakabayashi, S., Analytic smoothing effect for Schrödinger type equations with variable coefficients, in: Direct and Inverse Problems of Mathematical Physics (Newark, DE 1997), Int. Soc. Anal. Comput. 5, Kluwer Acad. Publ. (Dordracht, 2000), 185-219.

[KRY] Kapitanski, L., Rodnianski, I., Yajima, K., On the fundamental solution of a perturbed harmonic oscillator, Topol. Methods Nonlinear Anal. 9 (1997), 77-106.

[KaSa] Kapitanski, L., Safarov, Y., Dispersive smoothing for Schrödinger equations, Math. Res. Letters 3 (1996), 77-91.

[KaTa] Kato, K., Taniguchi, K., Gevrey regularizing effect for nonlinear Schrödinger equations, Osaka J. Math 33 (1996), 863-880.

[KaYa] Kato, T., Yajima, K., Some examples of smooth operators and the associated smoothing effect, Rev. Math. Phys. 1 (1989), 481-496.

[KPV] Kenig, C., Ponce, G., Vega, L., Oscillatory integrals and regularity of dispersive equations, Ind. Univ. Math. J. 40 (1991), 33-69.

[Ma1] Martinez, A., Estimates on complex interactions in phase Space, Math. Nachr. 167 (1994), 203-254.

[Ma2] Martinez, A., An Introduction to Semiclassical and Microlocal Analysis, UTX Series, Springer-Verlag New-York, 2002.

[MRZ] Morimoto, Y., Robbiano, L., Zuily, C., Remark on the smoothing for the Schrödinger equation, Indiana University Mathematic Journal 49 (2000), 1563-1579. 
[Na1] Nakamura, S.: On Martinez' method on phase space tunneling, Rev. Math. Phys. 7 (1995), 431-441.

[Na2] Nakamura, S., Propagation of the Homogeneous Wave Front Set for Schrödinger Equations, to appear in Duke Math. J. 126 (2005).

[Na3] Nakamura, S., Wave front set for solutions to Schrödinger equations, Preprint 2004

[RoZu1] Robbiano, L., Zuily, C., Microlocal analytic smoothing effect for Schrödinger equation, Duke Math. J. 100 (1999), 93-129.

[RoZu2] Robbiano, L., Zuily, C., Effet régularisant microlocal analytique pour l'équation de Schrödinger: le cas des données oscillantes, Comm. Partial Differential Equations 100 (2000) 1891-1906.

[RoZu3] Robbiano, L., Zuily, C., Analytic theory for the quadratic scattering wave front set and application to the Schrödinger equation, Soc. Math. France, Astérisque 283 (2002), 1-128.

[Sjl] Sjölin, P., Regularity of solutions to the Schrödinger equations, Duke Math. J. 55 (1987), 699-715.

[Sjs] Sjöstrand, J., Singularités analytiques microlocales, Soc. Math. France, Astérisque 95 (1982), 1-166.

[Wu] Wunsch, J., Propagation of singularities and growth for Schrödinger operators, Duke Math. J. 98 (1999), 137-186.

[Yaj1] Yajima, K., On smoothing property of Schrödinger propagators. Functional-Analytic Methods for Partial Differential Equations, Lecture Notes in Math., 1450 (1990), 20-35, Springer, Berlin.

[Yaj2] Yajima, K., Schrödinger evolution equations and associated smoothing effect, Rigorous Results in Quantum Dynamics, World Sci. Publishing, River Edge, NJ, 1991, 167-185.

[Yam] Yamazaki, M., On the microlocal smoothing effect of dispersive partial differential equations, Algebraic Analysis Vol. II, 911-926, Academic Press, Boston, MA,1988.

[Ze] Zelditch, S., Reconstruction of singularities for solutions of Schrödinger equation, Comm. Math. Phys. 90 (1983), 1-26. 